\title{
Dietary intake of food bioactives in a cohort of European adults with metabolic syndrome enrolled in the Pathway-27 trial
}

\author{
S. Sutulic ${ }^{1}$, M.J. Holmes ${ }^{1}$, S. Garelli ${ }^{2}$, M. Herrmann ${ }^{3}$, S. Seifert ${ }^{3}$, J. Amat ${ }^{4}$, A. Blot ${ }^{5}$, \\ E. Marcato ${ }^{2}$, C. Malpuech-Brugère ${ }^{4}$, A. Bub ${ }^{3}$, L. Ricciardiello ${ }^{2}$, A. Bordoni ${ }^{6}$ and C. Orfila ${ }^{1}$ \\ ${ }^{1}$ School of Food Science and Nutrition, University of Leeds, Leeds, LS2 9JT, UK, ${ }^{2}$ Department of Medical and \\ Surgical Sciences (DIMEC), University of Bologna, Italy, ${ }^{3}$ Max Rubner-Institut, Department of Physiology and \\ Biochemistry of Nutrition, Karlsruhe, Germany, ${ }^{4}$ Université Clermont Auvergne, INRA, UNH, Unité de Nutrition \\ Humaine, CRNH Auvergne, F-63000 Clermont-Ferrand, France, ${ }^{5} \mathrm{CHU}$ Clermont Ferrand, CRNH Auvergne, \\ F-63000 Clermont-Ferrand, France and ${ }^{6}$ Department of Agro-Alimentary Sciences and Technologies (DISTAL), \\ University of Bologna, Italy.
}

Metabolic syndrome (MetS) is the name given to a cluster of conditions that occur together more often than can be explained by chance. It is estimated that $20-25 \%$ of the global adult population have MetS $\mathrm{S}^{(1)}$ and are three times as likely to suffer a heart attack or stroke and have a fivefold greater risk of developing type-2 diabetes compared with people who do not have MetS ${ }^{(2-3)}$.

The PATHWAY-27 project evaluated the effectiveness of bioactive enriched food (BEF) on improving risk factors of MetS in participants at risk, or with, MetS. Following ethical approval, the trial was registered on clinicaltrials.gov (trial \# NCT02702713). The bioactive compounds selected were docosahexaenoic acid (DHA), oat beta-glucan (OBG) and grape anthocyanins (AC). In a multicentre, randomized, double-blind, nutritional intervention trial, 251 human volunteers were recruited to consume BEF for 12 weeks. Metabolic and anthropometric markers were measured at 0,6 and 12 weeks. Dietary exposure to DHA, OBG and AC from the participants' typical diet was assessed at baseline using a food frequency questionnaire. Participants were asked to estimate the frequency of consumption and portion size of foods known to contain each bioactive over 6-weeks prior to the start of the trial. Bioactive composition information was derived from national and international databases (EuroFIR; Polyphenol Explorer), literature information and direct analysis of foods. A deterministic exposure analysis was used to calculate dietary exposure for each participant enrolled in the trial according to equation (1) where $E=$ exposure of individual (j); $n=$ number of portions on day ( $k$ ) of food commodity (1), $w=$ portion of food, $c=$ concentration of bioactive.

The results (table 1) indicated generally low intakes of DHA, OBG and AC (evidenced by low mean) with great individual variation, and were associated with generally low consumption of fish, wholegrain oat, fruits, vegetables and legumes. Poor diet may contribute to the participants MetS phenotype and justifies interventions to increase bioactive intake. Bioactive exposure will be validated with metabolomics analysis of urine and serum.

$$
E_{\mathrm{j}}=\sum_{l=1}^{n(k)} w_{j k l} c_{j k l}
$$

Table 1. Bioactive dietary exposure at baseline of participants of the Pathway-27 trial $(n=281)$.

\begin{tabular}{|c|c|c|c|c|c|}
\hline & \multicolumn{5}{|c|}{ Bioactive exposure (mg/day) } \\
\hline & Mean & Std. Dev. & Std. Error & Min & $\operatorname{Max}$ \\
\hline DHA & 92.46 & $205 \cdot 60$ & $12 \cdot 98$ & $0 \cdot 00$ & $1659 \cdot 00$ \\
\hline OBG & $246 \cdot 28$ & $557 \cdot 63$ & $35 \cdot 48$ & $0 \cdot 00$ & $3330 \cdot 60$ \\
\hline $\mathrm{AC}$ & $79 \cdot 23$ & $101 \cdot 75$ & $7 \cdot 46$ & $0 \cdot 00$ & $685 \cdot 70$ \\
\hline
\end{tabular}

The PATHWAY-27 project is funded by the European Commission under the Seventh Framework Programme (Grant agreement number 311876). The authors thank Applications Santé des Lipides Sarl (France), Abro Biotec SL (Spain), Swedish Oat Fiber (Sweden), ADEXGO Kft. (Hungary) and Desarrollos Panaderos Levantinos SLL (Spain) for supplying ingredients and manufacturing bioactive enriched foods.

1. Alberti KGMM, Eckel RH, Grundy SM et al. (2009) Circulation 120(16), 1640-5.

2. Isomaa B, Almgren P, Tuomi T et al. (2001) Diabetes Care 24(4), 683.

3 Stern MP, Williams K, González-Villalpando C et al. (2004). Diabetes Care 27(11), 2676. 